\title{
A STUdY OF THE $K_{s}^{\circ} K_{s}^{\circ}$ SYSTEM IN THE REACTION $K^{-} p \rightarrow K_{s}^{\circ} K_{s}^{\circ} \Lambda$ AT $11 \mathrm{GeV} / \mathrm{c}^{\star}$
}

\author{
D. Aston,$^{1}$ N. AWAJi, ${ }^{a}$ T. BIEnZ, ${ }^{1}$ F. Bird, ${ }^{1}$ J. D'AMORE, ${ }^{3}$

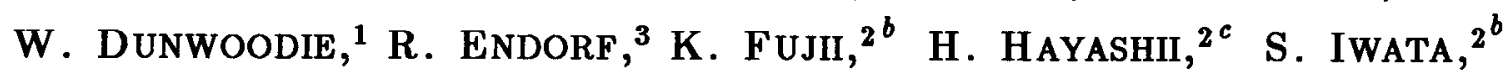 \\ W.B. Johnson, ${ }^{1}$ R. KaJikawa, ${ }^{2}$ P. Kunz, ${ }^{1}$ D.W.G.S. Leith, ${ }^{1}$ L. Levinson, ${ }^{d}$ \\ T. Matsui, ${ }^{2}$ B.T. Meadows, ${ }^{3}$ A. Miyamoto,${ }^{b}$ M. Nussbaum, ${ }^{3}$ H. OzaKi, ${ }^{2}$ \\ C.O. PaK, ${ }^{b}$ B.N. Ratcliff, ${ }^{1}$ D. Schultz, ${ }^{1}$ S. Shapiro, ${ }^{1}$ T. Shimomura, ${ }^{e}$ \\ P. K. Sinervo,${ }^{f}$ A. Sugiyama, ${ }^{2}$ S. Suzuki, ${ }^{2}$ G. Tarnopolsky,,$^{g}$ \\ T. TaUchI, ${ }^{b}$ N. Toge, ${ }^{1}$ K. UKaI, ${ }^{4}$ A. WaIte,${ }^{k}$ S. Williams ${ }^{i}$ \\ ${ }^{1}$ Stanford Linear Accelerator Center, Stanford University, \\ P.O. Box 4949 , Stanford, California 94305, U.S.A. \\ ${ }^{2}$ Department of Physics, Nagoya University, Furo-cho, Chikusa-ku, Nagoya 464, Japan \\ ${ }^{3}$ University of Cincinnati, Cincinnati, Ohio 15221, U.S.A. \\ ${ }^{4}$ Institute for Nuclear Study, University of Tokyo, Midori-cho, Tanashi, Tokyo 188, Japan

* Work supported in part by the Department of Energy under contract No. DE-AC03-76SF00515; the National Science Foundation under grant Nos. PHY82-09144, PHY85-13808, and the Japan U.S. Cooperative Research Project on High Energy Physics. Present Addresses :

a Fujitsu Limited, Nakahara-ku, Kawasaki 211, Japan.

b National Laboratory for High Energy Physics, KEK, Oho-machi, Tsukuba, Ibaraki 305, Japan.

c Nara Women's University, Kitauoya-nishi-machi, Nara-shi, Nara 630, Japan.

d Weizmann Institute, Rehovot 76100, Israel.

e Nippon BIO-RAD Laboratories, 1-2-7 Shiba-Daimon, Minato-ku, Tokyo 105, Japan.

$f$ University of Pennsylvania, Philadelphia, Pennsylvania 19104, U.S.A.

$g$ Hewlett-Packard Laboratories, 1501 Page Mill Road, Palo Alto, California 94304, U.S.A.

$h$ Department of Physics, University of Victoria, Victoria BC, Canada V8W 2Y2

i Diasonics Corp., 533 Cabot Rd., S. San Francisco, CA 94090, U.S.A. 


\section{ABSTRACT}

A 4.1 event/nb sample of $11 \mathrm{GeV} / \mathrm{c} K^{-} p$ interactions in the LASS spectrometer at SLAC has been used in an analysis of the exclusive $K_{s}^{\circ} K_{s}^{\circ} \Lambda$ final state. The $K_{s}^{\circ} K_{s}^{\circ}$ mass distribution exhibits a small cluster of events near threshold, but the mass spectrum is dominated by the $f_{2}^{\prime}(1525)$, which an amplitude analysis shows to be produced by both natural and unnatural parity exchange. This analysis also provides evidence for an $S$-wave state in the $f_{2}^{\prime}(1525)$ region, while production of the $f_{2}(1720)$ is not observed. However, there is structure at $\sim 2.2 \mathrm{GeV} / \mathrm{c}^{2}$ which is similar in shape to that observed in the region of the $X(2220)$ in radiative $J / \psi$ decay; an analysis of the angular distribution indicates a spin of at least 2 in this region. This, together with results from other analyses, indicates that the $X(2220)$ may be the $2^{++}$or $4^{++}$, mainly $s \bar{s}$, state expected near this mass in the conventional quark model. 


\section{Introduction}

This paper presents the results of an analysis of the $K_{s}^{\circ} K_{s}^{\circ} \Lambda$ final state produced in the hypercharge exchange reaction

$$
K^{-} p \rightarrow K_{s}^{\circ} K_{s}^{\circ} \Lambda
$$

where the final state particles decay in the charged mode so that the data sample contains only events for which the final state is fully reconstructed. The data are derived from an exposure of the LASS [1] spectrometer at SLAC to an $11 \mathrm{GeV} / \mathrm{c} \mathrm{K}^{-}$ beam. The sensitivity is 4.1 events/nb and the acceptance is rather uniform over the entire final state phase space.

In contrast to production in a pion beam, where $u \bar{u}$ and $d \bar{d}$ states dominate, $K^{-}$ beam hypercharge exchange channels are ideal for the study of the $s \bar{s}$ sector. The $K_{s}^{\circ} K_{s}^{\circ} \Lambda$ channel has several advantages over channels such as $K^{-} K^{+} \Lambda$ and $K_{s}^{\circ} K^{ \pm} \pi^{\mp} \Lambda$. There is no diffractive background, and the requirement that all three neutral particles from the primary vertex decay visibly makes the events readily distinguishable. Fits making use of kinematic constraints at the primary and all secondary vertices result in a data sample which has little contamination and good resolution. In addition, no further particle identification information is required, so no holes in the acceptance of the spectrometer are created. Finally, since the $K_{s}^{\circ} K_{s}^{\circ}$ system has even C-parity and, consequently, even angular momentum and parity, only meson states with $J^{P C}$ in the series $0^{++}, 2^{++}, 4^{++} \ldots$ are produced in reaction (1). This simplifies the spinparity analysis of the $K_{s}^{\circ} K_{s}^{\circ}$ system and compensates to some extent for the rather low statisics of the data sample. The extension of the $f_{2}^{\prime}(1525)$ trajectory [2] to higher spin states, $4^{++}$in particular, and the status of $0^{++}$states in the vicinity of $K \bar{K}$ threshold $[3,4]$ and at higher mass are matters of particular interest. Evidence for the $f_{2}(1720)[5]$ and $X(2220)[6]$ would be of great significance, since their production via hypercharge exchange in reaction (1) would cast doubt on their status as candidate glueball states.

After a brief description of the spectrometer, the data processing procedures and the characteristics of the resulting sample of events corresponding to reaction (1) 
are discussed. The results of a spherical harmonic moments analysis are then presented, and related to physical-region production amplitudes, the physics implications of which are considered.

\section{Experimental Procedure}

The experiment was carried out in SLAC beam line 20-21. Kaons in the $11 \mathrm{GeV} / \mathrm{c}$ $\mathrm{RF}$-separated beam, which had a typical $K / \pi$ ratio of $60: 1$, were identified by means of two threshold Čerenkov counters and defined in momentum, position and direction by a set of hodoscopes and proportional chambers. The residual level of beam impurity was measured to be less than $10^{-4}$. The beam then entered a liquid hydrogen target $84.6 \mathrm{~cm}$ in length, placed on the axis of the spectrometer at its upstream end.

The Large Aperture Superconducting Solenoid (LASS) spectrometer [1], shown in fig. 1 , consisted of a solenoidal vertex detector coupled to a forward dipole spectrometer. This configuration yielded $\sim 4 \pi$ coverage for charged particles and provided good momentum and vertex resolution. Charged particles produced in the target followed helical trajectories in the solenoid's $22.4 \mathrm{kG}$ field and were detected by six cylindrical proportional chambers surrounding the target, and nine planar proportional chambers covering the forward region. The six cylindrical chambers, and three of the planar chambers, were equipped with analogue cathode-strip readout as well as digital anode-wire readout [7] which enabled accurate and efficient determination of space points. The other chambers in the solenoid each consisted of three planes of anode wires. The high-flux central region of each of the the three planar cathode readout chambers was deadened with a mylar plug. Three small "plug" proportional chambers, each with three planes having $1 \mathrm{~mm}$ wire spacing, were installed to cover these insensitive regions.

The momentum determination for high momentum, low angle charged particles was enhanced by the dipole spectrometer. This spectrometer consisted of a dipole magnet with field integral $30 \mathrm{kG} \cdot \mathrm{m}$ along the axis and seven sets of magnetostrictive readout planar spark chambers (deactivated in the beam region), three of which were placed upstream of the dipole and four downstream. Hodoscopes and planar proportional chambers about the dipole were designed to identify spurious hits in the spark 
chambers by providing timing information. Two "plug" chambers upstream of the dipole covered the deadened beam region.

The experimental trigger was designed to accept $K^{-}$interactions yielding two or more charged particles in a box made up of the cylindrical and plug chambers which surrounded the target. Additional timing information from downstream scintillation hodoscopes was used to reduce the rate of spurious triggers. This multiplicity trigger was clean, efficient, and yielded almost uniform acceptance for the $K_{s}^{\circ} K_{s}^{\circ} \Lambda$ channel.

A detailed description of the LASS spectrometer can be found in ref. 1.

\section{Data Selection}

Approximately 113 million $K^{-}$triggers were recorded on $\sim 2200$ data tapes. These events were processed by a program which included track finding and fitting, beam reconstruction, secondary vertex finding, and event topology testing. This event reconstruction, which required the equivalent of $2.13081 \mathrm{~K}$ CPU-years, was shared between a processor farm of nine 168/E emulators at SLAC and the FACOM M200 and M382 computers at Nagoya University. The output was written to Data Summary Tapes (DST's) which contained reconstructed track parameters together with their associated hit coordinates and a list of acceptable candidate topologies connecting tracks with production and decay vertices.

The events on the DST's were filtered in several stages to select candidates for reaction (1). The initial selection required the events to have a manageable number of reconstructed tracks $(<10)$ and placed a loose limit on the missing longitudinal momentum. The events were then required to have three distinct reconstructed $V^{\circ}$ candidates consistent with two $K_{s}^{\circ}$ 's and a $\Lambda$ by imposing the invariant mass limits $0.447 \leq M_{\pi^{+} \pi^{-}} \leq 0.547 \mathrm{GeV} / \mathrm{c}^{2}$ for the $K_{s}^{\circ}$ candidates and $1.095 \leq M_{\pi^{-}} \leq 1.135$ $\mathrm{GeV} / \mathrm{c}^{2}$ for the $\Lambda$ candidates. The magnitudes of the missing transverse and longitudinal momenta were required to be less than 0.2 and $1.2 \mathrm{GeV} / \mathrm{c}$ respectively, and the missing mass squared against $K_{s}^{\circ} K_{s}^{\circ} \Lambda$ was required to satisfy $-0.04 \leq M M^{2} \leq$ $0.06\left(\mathrm{GeV} / \mathrm{c}^{2}\right)^{2}$. Limits were also placed on the quality of the primary and secondary vertices; in particular, the root mean square track-vertex separation for the vertex associated tracks was required to be less than $2 \mathrm{~cm}$. 
The 5663 events passing this selection were subjected to a geometrically constrained fit which took into account the effects of energy loss and multiple scattering. For each of the three $V^{\circ} \mathrm{s}$, the daughter tracks were refit to their associated coordinates such that they emanated from a common point, and such that the resultant momentum vectors for the three neutral tracks met at a common primary vertex point on the beam track. Events having no successful geometrically constrained fit were discarded.

The remaining events were subjected to a kinematically constrained fit which again involved fitting the individual tracks to their associated coordinates. In addition to the geometrical constraints used previously, this fit imposed four-momentum conservation at the primary and three secondary vertices, with the secondary vertices constrained to the nominal $K_{s}^{\circ}$ and $\Lambda$ masses. Events which yielded a converged fit to the $K_{s}^{\circ} K_{s}^{\circ} \Lambda$ hypothesis with a confidence level $\geq 10^{-6}$ were retained. This cut on the confidence level was chosen to keep a large fraction of the $K_{s}^{\circ} K_{s}^{\circ} \Lambda$ events while reducing contamination due to the final state $K_{s}^{\circ} K_{s}^{\circ} \Sigma^{\circ}$ as well as other sources of background. Monte Carlo studies showed that non $-K_{s}^{\circ} K_{s}^{\circ} \Lambda$ events tended to have very small confidence levels, if the fit converged at all.

Decay length cuts of $4 \mathrm{~mm}$ for $K_{s}^{\circ}$ and $2 \mathrm{~mm}$ for $\Lambda$ tracks were applied to remove remaining background events which accidentally satisfied the kinematic constraints. The data sample was restricted to correspond to forward meson production by requiring $t^{\prime} \leq 2.0(\mathrm{GeV} / \mathrm{c})^{2}$, with $t^{\prime}=|t|-|t|_{\min }$, where $t$ is defined as the 4-momentum transfer squared from the beam to the $K_{s}^{\circ} K_{s}^{\circ}$ system. The final data sample contains 441 events.

\section{Monte Carlo Studies and Acceptance}

Although the geometrical acceptance for events selected as described in section 3 proves to be essentially flat over the entire $4 \pi$ solid angle, acceptance corrections are necessary to account for the resolution and efficiency of detectors, decay and absorption of particles, and cuts applied in defining the sample. In addition, it must be demonstrated that distributions in lifetime, $V^{\circ}$ effective mass, momentum imbalance, missing mass, etc. of the selected data sample agree in shape with those for a similarly chosen sample of Monte Carlo events corresponding to reaction (1). 
To this end, Monte Carlo events simulating $K_{s}^{\circ} K_{s}^{\circ} \Lambda$ production were generated and the final state charged particles propagated through the spectrometer using the resolution and efficiency of the tracking devices and of the trigger as determined from the data. The Monte Carlo events were thrown flat in $M_{K}: K_{;}^{\circ}$ and in $t$-channel helicity angles, and with an exponential $t^{\prime}$ dependence of slope $2.3(\mathrm{GeV} / \mathrm{c})^{-2}$. Full event reconstruction was then performed by the DST production program used for the real data, producing Monte Carlo DST's in data format. These Monte Carlo events were then subjected to the same event selection and refitting procedures used for the real data. The acceptance at a particular point in the accessible $K_{s}^{\circ} K_{s}^{\circ}$ phase space was calculated as the ratio of the number of events surviving to the number of events thrown within a volume element surrounding this point. In this way, it was found that the acceptance for reaction (1) increases smoothly as a function of the mass of the $K_{s}^{\circ} K_{s}^{\circ}$ system from 0.23 at threshold to 0.28 at $2.6 \mathrm{GeV} / \mathrm{c}^{2}$, with very little dependence on $K_{s}^{\circ} K_{s}^{\circ}$ decay angles. It follows that the raw and acceptance-corrected $K_{s}^{\circ} K_{s}^{\circ}$ mass and decay angular distributions differ very little in shape.

As a demonstration of the effectiveness of the selection procedures outlined in section 3, Monte Carlo and data distributions in $V^{\circ}$ effective mass, $V^{\circ}$ proper lifetime, overall momentum balance and missing mass are compared in figs. $2-4$. For the purpose of these comparisons, the Monte Carlo events were weighted according to the observed $K_{s}^{\circ} K_{s}^{\circ}$ mass and decay angular distributions in order to take proper account of the dependence of resolution on these quantities. The resulting sum of weights was then normalized to the number of observed events.

Fig. 2a shows the $\pi^{+} \pi^{-}$invariant mass distribution for $K_{s}^{\circ}$ vertices in the geometrically constrained fits, and fig. $2 b$ shows the $\pi^{-} p$ mass for the $\Lambda$ vertices in the same events. The data points in figs. $2 c$ and $2 d$ show the corresponding distributions for the subset of events remaining in the final data sample, while the histograms represent the Monte Carlo results, which are in good agreement with the data distributions. In figs. $3 a$ and $3 b$ the ratio of the number of real events to the number of Monte Carlo events is shown as a function of proper decay length for the $K_{s}^{\circ}$ and $\Lambda$ vertices, respectively, in the final data sample. Again the data and Monte Carlo distributions are consistent.

The missing longitudinal momentum for events in the final data sample, using 
the results of the geometrically constrained fit, is plotted in fig. $4 a$ (solid dots). The corresponding distribution in missing mass squared opposite the $K_{s}^{\circ} K_{s}^{\circ} \Lambda$ is shown in fig. $4 \mathrm{~b}$, and the distribution in missing mass opposite the $K_{s}^{\circ} K_{s}^{\circ}$ is shown in fig. $4 \mathrm{c}$. In each case the Monte Carlo distribution (histogram) is in excellent agreement with the data.

To check for contamination in this data set from the reaction $K^{-} p \rightarrow K_{s}^{\circ} K_{s}^{\circ} \Sigma^{\circ}$, Monte Carlo events of this reaction were generated, subjected to the data selection criteria, and fit as $K_{s}^{\circ} K_{s}^{\circ} \Lambda$. The $K_{s}^{\circ} K_{s}^{\circ} \Sigma^{\circ}$ final state could be a significant source of background as it is topologically indistinguishable from that of reaction (1), it would yield very similar distributions to those shown in figs. $2-4$, and the undetected gamma would have a rather low momentum in the lab, since the $K_{s}^{\circ} K_{s}^{\circ}$ system is peripherally produced. However, only $\sim 15 \%$ of these events yielded a good, kinematically constrained fit, mainly because the missing gamma carried away an amount of transverse momentum which was large compared to the observed transverse momentum imbalance for events from reaction (1). The missing $p_{T}$ is shown for the final data sample (points), and for the $K_{s}^{\circ} K_{s}^{\circ} \Lambda$ Monte Carlo data (open histogram), in fig. 5a. The two distributions are consistent with each other. Fig. $5 \mathrm{~b}$ shows the missing transverse momentum of the $K_{s}^{\circ} K_{s}^{\circ} \Sigma^{\circ}$ generated events, from the results of the geometrically constrained fit, before (open) and after (shaded) requiring a successful kinematically constrained fit to the $K_{s}^{\circ} K_{s}^{\circ} \Lambda$ hypothesis. The number of events has been normalized using an estimated relative cross section for $\Sigma^{\circ}$ and $\Lambda$ production of $30 \pm 10 \%[8,9]$. From this comparison the $K_{s}^{\circ} K_{s}^{\circ} \Sigma^{\circ}$ contamination in the final sample was estimated to be $16 \pm 6$ events, or about $4 \%$.

It follows from this discussion that the analysis sample for reaction (1) is rather clean, and that the Monte Carlo program successfully models the characteristics of the spectrometer and the effects of the data processing and selection procedures. Consequently, the Monte Carlo data sample for reaction (1) may be used to estimate the mass dependence of the $K_{s}^{\circ} K_{s}^{\circ}$ mass resolution; this was found to be $\sim 5 \mathrm{MeV} / \mathrm{c}^{2}$ at threshold, and to increase linearly to $\sim 12 \mathrm{MeV} / \mathrm{c}^{2}$ at $2.5 \mathrm{GeV} / \mathrm{c}^{2}$. 


\section{General Features of the Data}

The uncorrected $K_{s}^{\circ} K_{s}^{\circ}$ mass distribution for the final sample of events corresponding to reaction (1) is shown in fig. 6. There is an accumulation of events near threshold, then a small bump in the $f_{2}(1270) / a_{2}(1320)$ region followed by a large peak resulting from the production of $f_{2}^{\prime}(1525)$. No $f_{2}(1720)$ signal is observed, but there does appear to be a broad enhancement in the region $\sim 2.0-2.3 \mathrm{GeV} / \mathrm{c}^{2}$.

The invariant mass distribution for the $K_{s}^{\circ} \Lambda$ system is shown in fig. 7. The distribution shows no resonant structure, and appears simply to reflect the features of the $K_{s}^{\circ} K_{s}^{\circ}$ spectrum. This is evident from the Dalitz plot (fig. 8).

The Chew-Low plot (fig. 9) demonstrates clearly the peripheral nature of the production of the $K_{s}^{\circ} K_{s}^{\circ}$ system. Almost all of the events with $K_{s}^{\circ} K_{s}^{\circ}$ mass less than $3 \mathrm{GeV} / \mathrm{c}^{2}$ are localized to the region $t^{\prime} \leq 2(\mathrm{GeV} / \mathrm{c})^{2}$, although there are a few events clustered along the large- $t$ boundary. These correspond to backward production of the $K_{s}^{\circ} K_{s}^{\circ}$ system in the center of mass; however they show no structure in mass and will not be considered further. The analysis sample discussed in the remainder of this paper is restricted to the events having $t^{\prime} \leq 2(\mathrm{GeV} / \mathrm{c})^{2}$.

Since the final state $\Lambda$ is reconstructed in this analysis, the polarization of the $\Lambda$ is measurable. The polarization, $P_{\Lambda}$, is defined as

$$
P_{\Lambda}=\frac{2}{\alpha} \cdot \frac{N_{+}-N_{-}}{N_{+}+N_{-}}
$$

where $\alpha(=.642)$ is the decay asymmetry parameter for the $\Lambda$, and $N_{+}\left(N_{-}\right)$is the number of events for which $\vec{p} \cdot \vec{n}$ is positive (negative); $\vec{p}$ is the decay proton momentum in the $\Lambda$ rest frame, and $\vec{n}$ is normal to the the $\Lambda$ production plane (defined by $\left.\vec{n}=\left(\vec{K}_{b e a m}^{-} \times \vec{\Lambda}\right)\right)$. Due to the small number of events in this analysis the uncertainties in the measured values of $P_{\Lambda}$ are large, and it is not possible to determine its behavior precisely. However, $P_{\Lambda}$ appears to be substantial in the 1.5 to 1.8 $\mathrm{GeV} / \mathrm{c}^{2} K_{s}^{o} K_{s}^{o}$ mass region (fig. 10a) and to be consistent with zero elsewhere. In the $f_{2}^{\prime}(1525)$ region $\left(1.4 \leq M_{K_{0}^{\circ} K_{:}^{\circ}} \leq 1.7 \mathrm{GeV} / \mathrm{c}^{2}\right)$, shown in fig. $10 \mathrm{~b}, P_{\Lambda}$ is close to maximal for $t^{\prime} \sim 0.2(\mathrm{GeV} / \mathrm{c})^{2}$, then decreases and becomes consistent with zero for $t^{\prime} \sim 0.5-1.0(\mathrm{GeV} / \mathrm{c})^{2}$. The polarization may become large again for $t^{\prime}>1.0(\mathrm{GeV} / \mathrm{c})^{2}$, 
but the number of events in this region is small and the corresponding measurement uncertainty is large.

Since the $K_{s}^{\circ} K_{s}^{\circ}$ system is peripherally produced it is natural to investigate its decay properties in the $t$-channel helicity (Gottfreid-Jackson) frame. For the events having $t^{\prime} \leq 2.0(\mathrm{GeV} / \mathrm{c})^{2}$, the polar $\left(\theta_{J}\right)$ and azimuthal $\left(\phi_{T Y}\right)$ angular distributions are shown as a function of $M_{K} K_{a}^{\circ}$ in fig. 11. Only the $K_{s}^{\circ}$ having $\cos \theta_{J} \geq 0$ contribute to fig. 11.

The six events having mass less than $1.05 \mathrm{GeV} / \mathrm{c}^{2}$ are distributed uniformly in $\cos \theta_{J}$ as would be expected for a $K_{s}^{\circ} K_{s}^{\circ}$ system in an S-wave state. The $\phi_{T Y}$ distribution, folded about $0^{\circ}$, is also rather uniform. At higher mass, the region up to $\sim 1.7 \mathrm{GeV} / \mathrm{c}^{2}$, which contains the strong $f_{2}^{\prime}(1525)$ band as well as the cluster of events at the $f_{2}(1270)$ and $a_{2}(1320)$, exhibits a forward peak whose shape is approximately quadratic in $\cos \theta_{J}$. The distribution then becomes rather isotropic in the range 1.7 to $2.4 \mathrm{GeV} / \mathrm{c}^{2}$ except for the development of a peak for $\cos \theta_{J} \geq 0.7$ for $K_{s}^{\circ} K_{s}^{\circ}$ mass greater than $\sim 2.0$ $\mathrm{GeV} / \mathrm{c}^{2}$. Above $2.4 \mathrm{GeV} / \mathrm{c}^{2}$ only the forward peak persists and becomes more localized to the forward direction as the mass increases.

The azimuthal symmetry observed in fig. $11 \mathrm{~b}$ is confirmation of parity conservation in the production and decay process. The depopulation at $\sim \pm 180^{\circ}$ and at $\sim 0^{\circ}$ for $K_{s}^{\circ} K_{s}^{\circ}$ mass less than $\sim 2.4 \mathrm{GeV} / \mathrm{c}^{2}$ indicates that helicity 1 natural parity exchange gives an important contribution to the $t$-channel production.

The mass dependence of the $K_{s}^{\circ} K_{s}^{\circ}$ angular distribution is discussed quantitatively in terms of spherical harmonic functions, $Y_{l m}\left(\cos \theta_{J}, \phi_{T Y}\right)$, in the next section.

\section{Spherical Harmonic Moments Analysis}

The acceptance-corrected angular distribution of the $K_{s}^{\circ} K_{s}^{\circ}$ system in the $t$ channel helicity frame can be written in terms of spherical harmonic functions as follows,

$$
I(X, \Omega)=\frac{1}{\sqrt{4 \pi}}\left[\sum_{l=0,2,4 \ldots} t_{l 0}(X) Y_{l 0}(\Omega)+\sum_{l=2,4 \ldots} \sum_{m=1}^{m_{\max }} t_{l m}(X) 2 \operatorname{Re}\left\{Y_{l m}(\Omega)\right\}\right]
$$




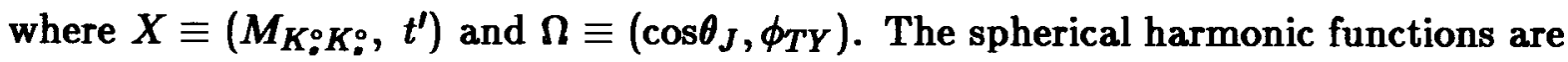
normalized such that

$$
\int_{0}^{4 \pi}\left[Y_{l 0}(\Omega)\right]^{2} \cdot d \Omega=1, \quad \int_{0}^{4 \pi}\left[\operatorname{Re}\left\{Y_{l m}(\Omega)\right\}\right]^{2} \cdot d \Omega=\frac{1}{2},
$$

and terms involving $\operatorname{Im}\left\{Y_{l m}(\Omega)\right\}$ vanish as a result of parity conservation. The summation is over only even $l$ values because of the indistinguishability of the two $K_{s}^{\circ}$, and it was found empirically that $m_{\max }$ need not be greater than 2 to provide a satisfactory description of the data. In this analysis, the data with $t^{\prime} \leq 2(\mathrm{GeV} / \mathrm{c})^{2}$ were divided into discrete bins in $M_{K_{\bullet}} K_{\bullet}^{\circ}$, and the acceptance-corrected spherical harmonic moments, $\mathbf{t}_{l m}(X)$, obtained for each bin.

The distribution observed in the data is $I(X, \Omega)$ modified by the acceptance and resolution of the spectrometer. These corrections were obtained using the Monte Carlo procedure described above, and the acceptance-corrected moments found from the moments of the data by the linear algebra (moments) method [10]. The results of other conventional methods, such as a $\log$-likelihood fit or a procedure in which each event was weighted according to the inverse of the local acceptance value, agreed with those of this method within errors.

Fig. 12 shows the acceptance-corrected $K_{s}^{\circ} K_{s}^{\circ}$ mass distribution ( $t_{00}$ moment) up to $3.6 \mathrm{GeV} / \mathrm{c}^{2}$. Because of the uniform nature of the acceptance, the features of this distribution are identical to those observed in fig. 6 for the raw data sample.

\subsection{The Threshold Region}

Inset in fig. 12 is the $K_{s}^{\circ} K_{s}^{\circ}$ threshold region plotted in $10 \mathrm{MeV} / \mathrm{c}^{2}$ bins; an accumulation of six events within the first $30 \mathrm{MeV} / \mathrm{c}^{2}$ out of only seven below $1.1 \mathrm{GeV} / \mathrm{c}^{2}$ can be seen. These six events are also localized to the $t^{\prime}$ region $0.13-0.25(\mathrm{GeV} / \mathrm{c})^{2}$, whereas the event with a $K_{s}^{\circ} K_{s}^{\circ}$ mass value of $1.083 \mathrm{GeV} / \mathrm{c}^{2}$ has a large $t^{\prime}$ value, viz. $0.85(\mathrm{GeV} / \mathrm{c})^{2}$. Their angular distributions, as discussed above, are essentially flat, and are consistent with the production of $S$-wave only ${ }^{\star)}$. In addition, a fit to the mass

*) From an analysis of the reaction $K^{-} p \rightarrow \phi \Lambda$ in the present experiment it is estimated that the decay $\phi \rightarrow K_{:}^{\circ} K_{t}^{\circ}$ with both kaons decaying to $\pi^{+} \pi^{-}$would yield less than 0.25 events in the threshold region. 
spectrum of fig. 12 in the range $1.15-1.80 \mathrm{GeV} / \mathrm{c}^{2}$ in terms of $f_{2}(1270)$ and $f_{2}^{\prime}(1525)$ production (see below) predicts a negligible $D$-wave intensity below $1.05 \mathrm{GeV} / \mathrm{c}^{2}$. Two $0^{++}$mesons (the $f_{0}(975)$ and the $a_{0}(980)$ ) which decay to $K \bar{K}$ are known to lie in the mass region just below $K \bar{K}$ threshold [2] and might be considered as the source of these events. Recently, Au et al. [3] have suggested the existence of an additional resonance coupling to $K \bar{K}$ in this mass region, which they propose as a candidate for a scalar glueball. The resonance positions and widths differ in their model from the more traditional ones, so in principle it is possible to test their model using the data presented here. In practice, the limited statistics make the effective discrimination rather weak. A fit of the events below $1.05 \mathrm{GeV} / \mathrm{c}^{2}$ to the form used by Flatté [11] gives a width of $22 \pm 12 \mathrm{MeV} / \mathrm{c}^{2}$ for a pole situated at $K_{s}^{\circ} K_{s}^{\circ}$ threshold (the precise value depends to some extent on details of the coupled channel and relative $K \bar{K}$ coupling). A pole on the real axis, such as the $S_{2}(988)$ suggested by Au et al., is consistent with the data and preferred at about the $90 \%$ confidence level over the conventional forms of the $f_{0}(975)$ or $a_{0}(980)$.

\subsection{THE $f_{2}^{\prime}(1525)$ REgION}

Above the threshold region the most prominent feature of the data is the production of the $f_{2}^{\prime}(1525)$. There is also a small peak just below $1.3 \mathrm{GeV} / \mathrm{c}^{2}$ which can be attributed to production of $f_{2}(1270)$ or $a_{2}(1320)$. In the region around $1.7 \mathrm{GeV} / \mathrm{c}^{2}$, several experiments studying the radiative decay of the $J / \psi[5]$ have reported observing the production of a $2^{++}$object, the $f_{2}(1720)$, with a strong $K \bar{K}$ decay mode. A comparison of the mass spectrum of fig. 12 with that from the analysis of the reaction $J / \psi \rightarrow K_{s}^{\circ} K_{s}^{\circ} \gamma$ by the Mark III collaboration [6] is shown in fig. 13; the LASS data have been scaled down by 0.125 to match the Mark III data at the $f_{2}^{\prime}(1525)$ peak. The relative strength of the $f_{2}^{\prime}(\mathbf{1 5 2 5})$ and $f_{2}(\mathbf{1 7 2 0})$ signals is clearly very different in the two reactions, and there is no evidence for the production of the $f_{2}(1720)$ in the present experiment.

The distribution of fig. 12 is replotted in bins of $0.04 \mathrm{GeV} / \mathrm{c}^{2}$ in fig. $14 \mathrm{~b}$ in direct comparison with data at $4.2 \mathrm{GeV} / \mathrm{c}[9]$ and $40 \mathrm{GeV} / \mathrm{c}[12]$; clearly, the mass spectra are very similar. The curve shown on each distribution of fig. 14 corresponds to a fit of the incoherent sum of Breit-Wigner line-shapes describing the $f_{2}(1270)$ and 
the $f_{2}^{\prime}(1525)$ resonances in the range $1.16-1.8 \mathrm{GeV} / \mathrm{c}^{2}$; in each case an additional incoherent $a_{2}(1320)$ contribution was not required by the fit. The expression used is

$$
\frac{d \sigma}{d M}=M \cdot \sum_{r} c_{r}\left|B W_{r}\right|^{2}
$$

where $B W_{r}$ is a Breit-Wigner line-shape with parametrization

$$
\begin{aligned}
B W_{r} & =\frac{\sqrt{\Gamma_{f}^{r}}}{M_{r}^{2}-M^{2}-i M_{r} \Gamma_{t o t}^{r}} \\
\Gamma_{f}^{r} & =\Gamma_{0 f}^{r}\left(\frac{q}{q_{r}}\right)^{2 l+1}\left(\frac{M_{r}}{M}\right) \\
\Gamma_{t o t}^{r} & =\sum_{f} \Gamma_{f}^{r} ;
\end{aligned}
$$

$M$ is the invariant mass of the $K_{s}^{\circ} K_{s}^{\circ}$ system, the $M_{\mathrm{r}}$ are the masses of the resonances, and the $c_{r}$ are constants; $q$ is the $K_{s}^{\circ}$ momentum in the $K_{s}^{\circ} K_{s}^{\circ}$ rest frame, and $q_{r}$ its value at resonance; $\Gamma_{0 f}^{r}$ is the partial width of resonance $r$ to final state $f$. The mass and width of the $f_{2}(1270)$ arc fixcd at their nominal values [2], and the parameters of the $f_{2}^{\prime}(1525)$ determined by the fit.

The results obtained for the data from the present experiment (fig. 14b) are

$$
\begin{aligned}
M_{r} & =1526.8 \pm 4.3 \mathrm{MeV} / \mathrm{c}^{2} \\
\Gamma_{0}^{r} & =90.2 \pm 11.8 \mathrm{MeV} / \mathrm{c}^{2}
\end{aligned}
$$

At $4.2 \mathrm{GeV} / \mathrm{c}[9]$

$$
\begin{aligned}
M_{r} & =1526.7 \pm 5.5 \mathrm{MeV} / \mathrm{c}^{2} \\
\Gamma_{0}^{r} & =79.3 \pm 15.7 \mathrm{MeV} / \mathrm{c}^{2}
\end{aligned}
$$

while at $40 \mathrm{GeV} / \mathrm{c}[12]$

$$
\begin{aligned}
M_{r} & =1521.7 \pm 7.5 \mathrm{MeV} / \mathrm{c}^{2} \\
\Gamma_{0}^{r} & =70.4 \pm 22.4 \mathrm{MeV} / \mathrm{c}^{2}
\end{aligned}
$$

The weighted averages are

$$
\begin{aligned}
M_{r} & =1525.9 \pm 3.1 \mathrm{MeV} / \mathrm{c}^{2} \\
\Gamma_{0}^{r} & =83.9 \pm 8.7 \mathrm{MeV} / \mathrm{c}^{2}
\end{aligned}
$$

and the curve of fig. 12 was obtained from a fit to the data with the $f_{2}^{\prime}(1525)$ parameters 
fixed to these values. The resulting cross section for the reaction $K^{-} p \rightarrow f_{2}^{\prime}(1525) \Lambda$ at $11 \mathrm{GeV} / \mathrm{c}$ after all corrections $[2]$ is $3.25 \pm 0.28 \mu b$.

This value is compared with those at other beam momenta $[8,9,12,13,14]$ in fig. 15 . In this figure, data from experiments which have no separation of recoil $\Lambda / \Sigma[12,14]$ have been corrected to recoil $\Lambda$ by the scale factor of $0.79 \pm 0.09$ estimated from ref. 9 . In addition, the low statistics bubble chamber values at 10 and $16 \mathrm{GeV} / \mathrm{c}$ have been reduced by $20 \%$ to allow for background under the $f_{2}^{\prime}(1525)$. The original cross section value for the reaction (1) at $4.2 \mathrm{GeV} / \mathrm{c}$ quoted in ref. 9 is smaller than that plotted in fig. 15. In the original paper the spectrum was parametrized by a polynomial for the background and a Breit-Wigner for the $f_{2}^{\prime}(1525)$. The result was that $\sim 20 \%$ of the $f_{2}^{\prime}(1525)$ signal estimated according to the fit shown in fig. 14 a was assigned to background. The cross section corresponding to the present parametrization is $15.8 \pm$ $2.5 \mu b$. The line shown in fig. 15 connects this value to that obtained at $11 \mathrm{GeV} / \mathrm{c}$ in the present experiment. Clearly it provides a good description of the energy dependence of the other measurements, and, in particular, extrapolates with good accuracy to 40 $\mathrm{GeV} / \mathrm{c}[12]$.

The fit to the distribution of fig. 12 yields a $K \bar{K}$ channel cross section of $f_{2}(1270)$ of $0.57 \pm 0.15 \mu b$ for the reaction $K^{-} p \rightarrow\left(f_{2}(1270) \rightarrow K \bar{K}\right) \Lambda$.

An additional $f_{2}(1720)$ Breit-Wigner term in the expression used to describe the data of fig. 12 is rejected by the fit. The corresponding upper limit (at $95 \%$ c.l.) on the production of $f_{2}(1720)$ and subsequent decay to $K \bar{K}$ is estimated to be $94 n b$.

The acceptance-corrected moments of the $K_{s}^{\circ} K_{s}^{\circ}$ system are shown as a function of mass in fig. 16 for the region above $1.1 \mathrm{GeV} / \mathrm{c}^{2}$; the moments with $l=6$ and $l=8$ are included for masses greater than $1.75 \mathrm{GeV} / \mathrm{c}^{2}$, but are consistent with zero throughout this range. Clear peaks corresponding to the $f_{2}^{\prime}(1525)$ are seen in the $t_{20}$ and $t_{42}$ moments. The significance of the $\mathrm{m}=2$ moment indicates a substantial contribution to the production from natural parity exchange in the $t$-channel. Fig. 17 shows the $t^{\prime}$ dependence of the acceptance-corrected moments in the region $1.4 \leq M_{K}: K_{\bullet}: 1.7$ $\mathrm{GeV} / \mathrm{c}^{2}$. These moments are used in section 7 to determine the amplitude structure of the $f_{2}^{\prime}(\mathbf{1 5 2 5})$ differential cross section. 


\subsection{THE $2.2 \mathrm{GeV} / \mathrm{c}^{2}$ Region}

In fig. 18a, the mass spectrum from the analysis of the reaction $J / \psi \rightarrow K_{s}^{\circ} K_{s}^{\circ} \gamma$ by the Mark III collaboration [6] is compared to that of fig. 12; the LASS data in the region 1.8-2.7 GeV/c $\mathrm{c}^{2}$ have been normalized to the number of Mark III events in this same interval. The distributions are quite similar, in particular near $2.2 \mathrm{GeV} / \mathrm{c}^{2}$ where the $X(2220)$ has been observed. In this same region, the $\mathbf{t}_{20}$ moment differs significantly from zero (fig. 16). More explicitly, the distribution in $\cos \theta_{J}$ for events in the interval 2.0-2.4 GeV/c $\mathrm{c}^{2}$ is as shown in fig. 18b; the forward peak signals the presence of amplitudes with $J \geq 2$. The peaking effect is more pronounced for the events in the region $2.15-2.3 \mathrm{GeV} / \mathrm{c}^{2}$ (shaded histogram), indicating its association with the $X(2220)$. Preliminary results for the $K^{+} K^{-}$system produced via the reaction $K^{-} p \rightarrow K^{+} K^{-} \Lambda$ in the present experiment [15] exhibit a small peak in the moments $t_{10}-t_{80}$ in the vicinity of $2.2 \mathrm{GeV} / \mathrm{c}^{2}$. In addition, for the reaction $\pi^{-} p \rightarrow \eta \eta^{\prime} n$ at 38 and $100 \mathrm{GeV} / \mathrm{c}[16]$ a small but narrow peak has been observed in $M_{\eta \eta^{\prime}}$ at $\sim 2.2 \mathrm{GeV} / \mathrm{c}^{2}$ in association with a highly anisotropic angular distribution.

These results suggest that the $X(2220)$ may be an ordinary, mainly $s \bar{s}$, hadron, rather than an exotic state as has been previously proposed. Since the experimental evidence is that the spin is at least 2 , and since a spin 6 state would not be expected below $\sim 2.5 \mathrm{GeV} / \mathrm{c}^{2}$, the only viable quantum numbers for such a hadron coupling to $K_{s}^{\circ} K_{s}^{\circ}$ are $J^{P C}=2^{++}$or $4^{++}$. Mainly $s \bar{s}$ states with these quantum numbers are expected to exist at $\sim 2.2 \mathrm{GeV} / \mathrm{c}^{2}$ as members of the ${ }^{3} F$ ground state in the quark model [17]. However, the present angular distribution data are not so precise that a unique $J^{P C}$ assignment can be made.

It follows that the $X(2220)$ may well correspond to the first recurrence of the $f_{2}^{\prime}(1525)$, or of the $0^{++}$state discussed in subsection 7.2 below. 


\section{Amplitude Decomposition}

The moments, $t_{l m}$, of equation (2) can be expressed as bilinear products of amplitudes, $L_{\lambda \pm}$, for production of a $K_{s}^{\circ} K_{s}^{\circ}$ system of spin $L$ and $t$-channel helicity $\lambda$ via natural $(+)$ or unnatural( $(-)$ parity exchange. Given the limited statistical level of the data, a number of simplifying assumptions have been made in the analysis described here. Each term corresponds to a sum over the helicity flip and non-flip amplitudes at the baryon vertex and the isospin of the $K_{s}^{\circ} K_{s}^{\circ}$ system. It has been assumed that amplitudes with the same naturality are coherent, that averaging over a wide range in $t^{\prime}$ is permitted, that $L_{\lambda \pm}=0$ for $\lambda>1$, and that there are no significant amplitudes beyond the $D$-wave. With these restrictions, the moments calculated in each $M_{K}: K$ : bin are related to the amplitudes by

$$
\begin{aligned}
& \mathbf{t}_{00}=S_{0}^{2}+D_{0}^{2}+D_{+}^{2}+D_{-}^{2} \\
& \mathbf{t}_{20}=0.639 D_{0}^{2}+0.319\left(D_{-}^{2}+D_{+}^{2}\right)+2 S_{0} D_{0} \\
& \mathbf{t}_{21}=1.414 S_{0} D_{-}+0.452 D_{0} D_{-} \\
& \mathbf{t}_{22}=0.391\left(D_{-}^{2}-D_{+}^{2}\right) \\
& \mathbf{t}_{40}=0.857 D_{0}^{2}-0.571\left(D_{-}^{2}+D_{+}^{2}\right) \\
& \mathbf{t}_{41}=1.107 D_{0} D_{-} \\
& \mathbf{t}_{42}=0.452\left(D_{-}^{2}-D_{+}^{2}\right)
\end{aligned}
$$

where the simplified notation, $L_{0} \equiv L_{0-}, L_{ \pm} \equiv L_{1 \pm}, L^{2} \equiv|L|^{2}$, and $L_{1} L_{2} \equiv \operatorname{Re}\left(L_{1} L_{2}^{*}\right)$ has been used.

The relations above were used to fit the moduli of the amplitudes and their relative phases to the measured moments of the data. In general, two solutions to these coupled quadratic equations are obtained, which, within the limited statistics, provide an equally good description of the data. When this ambiguity occurs in the present analysis, however, the solutions for the moduli are very close to each other and lead to similar conclusions. The relative phases are not well determined by the fits, and so will not be presented.

The resulting intensity distributions for the individual amplitudes are shown as a function of mass in figs. 19 and 20a, and as a function of $t^{\prime}$ in fig. 22. In those intervals 
having two solutions, the cross symbol denotes the solution yielding the smaller Swave amplitude, and the distinct solutions differ usually by less than one standard deviation; the error bars shown represent the limits of the one sigma $\chi^{2}$ contour for each bin.

\subsection{THE D-WAVE}

The $D_{0}$ and $D_{+}$amplitudes (fig. 19) show similar contributions to the production of the $f_{2}(1270) / a_{2}(1320)$ and the $f_{2}^{\prime}(1525)$, while the $D_{-}$amplitudes are small and exhibit no structure. The fit shown in fig. $19 \mathrm{~b}$ is to the sum of the $D_{0}$ and $D_{+}$ intensities in the 1.4 to $1.7 \mathrm{GeV} / \mathrm{c}^{2}$ mass range using a single Breit-Wigner form for the $f_{2}^{\prime}\left(\mathbf{1 5 2 5 )}\right.$. For the mass intervals yielding two solutions, the $\chi^{2}$ contour is modeled by a double parabola with minima at the smaller and larger solutions, and with widths to the lower and higher sides corresponding to the error bars shown in the figure. The resulting parameter values for the $f_{2}^{\prime}(1525)$ are

$$
\begin{aligned}
M_{D} & =1527.4_{-10.5}^{+14.7} \mathrm{MeV} / \mathrm{c}^{2} \\
\Gamma_{D} & =88.3_{-20.4}^{+47.5} \mathrm{MeV} / \mathrm{c}^{2}
\end{aligned}
$$

clearly these are consistent with those obtained in section 6 from the simple fit to the mass spectrum.

The cross section for $f_{2}^{\prime}(1525)$ production in the reaction $K^{-} p \rightarrow f_{2}^{\prime}(1525) \Lambda$ determined by integrating this fit from 1.35 to $1.70 \mathrm{GeV} / \mathrm{c}^{2}$ and correcting for unseen decays [2] is found to be $2.16 \pm 0.20 \mu b$, of which $\sim 60 \%$ corresponds to the $D_{+}$ amplitude. Inclusion of the $D_{-}$contribution increases the total $D$-wave cross section to $\sim 2.5 \mu b$. The remaining discrepancy between this value and that quoted in section 6 as the $f_{2}^{\prime}(1525)$ cross section, viz. $\sim 0.75 \mu b$, is due to the presence of the S-wave contribution shown in fig. 20a. 


\subsection{ThE S-WAVE}

In the absence of an $S$-wave amplitude, $t_{21} \cong 0.4 \mathbf{t}_{41}$. The distributions of figs. 16 and 17 demonstrate that this relationship is not satisfied in the vicinity of the $f_{2}^{\prime}(1525)$, and hence that the $\mathrm{S}$-wave amplitude is non-zero in this region. This is shown explicitly in fig. 20a, and although the uncertainties are large, it is clear that an S-wave contribution is indeed present. In order to define the significance of the S-wave amplitude, an additional fit to a single mass interval from $1.4-1.7 \mathrm{GeV} / \mathrm{c}^{2}$ was performed. This fit yields two solutions with identical $\chi^{2}$. The dependence of $\chi^{2}$ on $\left|S_{0}\right|$ in the vicinity of the minima is shown in fig. 21 , and it is clear that even the smaller amplitude solution differs from zero by more than four standard deviations. It should also be noted that although the $\chi^{2}$ distribution is approximately symmetric about the minima when plotted as a function of $\left|\mathrm{S}_{0}\right|$ (fig. 21a), it exhibits highly asymmetric behavior with respect to $\left|S_{0}\right|^{2}$ (fig. 21b). It follows that the error bars, e.g. in fig. 20a, must be interpreted with some care when the significance of the $S_{0}$ amplitude is assessed.

From fig. 21, the 95\% confidence level limits on the S-wave cross section times branching ratio to $K \bar{K}$ are

$$
0.08 \mu b<\sigma\left(K^{-} p \rightarrow(\mathrm{S}-\text { wave }) \Lambda\right) \cdot B R((\mathrm{~S}-\text { wave }) \rightarrow K \bar{K})<2.1 \mu b
$$

and the cross section corresponding to the average of the two solutions is $\sim 0.75 \mu b$.

The S-wave intensity distribution of fig. 20a is very similar to that obtained from an analysis of the $K \bar{K}$ system produced in the reaction $K^{-} p \rightarrow K^{+} K^{-} \Lambda$ at $8.25 \mathrm{GeV} / \mathrm{c}$ [18]. This is illustrated in fig. $20 \mathrm{~b}$, which corresponds to the sum of the helicity flip and non-flip S-wave intensities of ref. 18. These independent results are very similar and suggest the existence of a $0^{++}$state, the $f_{0}^{\prime}$, approximately degenerate in mass and width with the $f_{2}^{\prime}(1525)$. This is shown explicitly in fig. 20a by means of the dashed curve which is simply that of fig. $19 \mathrm{~b}$ scaled down by a factor of three.

A partial wave analysis of the $K \bar{K} \pi$ system produced in the reaction $K^{-} p \rightarrow$ $K_{s}^{\circ} K^{ \pm} \pi^{\mp} \Lambda$ in the present experiment [19], has confirmed the existence of the $1^{++}$ $f_{1}^{\prime}(1525)$ (the $D^{\prime}$ ) claimed in an earlier analysis of these reactions at $4.2 \mathrm{GeV} / \mathrm{c}$ [20]. It follows that the $0^{++}$suggested above, together with the $f_{2}^{\prime}$ and the $f_{1}^{\prime}$ would form the 
mainly $s \bar{s}$ triplet of isoscalar states expected in the quark model. The approximate mass degeneracy of these states would then imply that the spin-orbit interaction in the strangeonium sector is rather weak in comparison to that predicted, for example, in ref.17. Furthermore, this $0^{++}$state would replace the $f_{0}(975)$ as the ${ }^{3} P_{0}$ ground state. This leads to the inference that the $f_{0}(975)$ is not a conventional $q \bar{q}$ state, and that it may well be a weakly bound $K \bar{K}$ system as suggested by Weinstein and Isgur [21]. In these papers, it was suggested that the $a_{0}(980)$ might also be such a state. If this is the case, and if the spin-orbit interaction in the isovector sector is also weak, then the missing $0^{++}$isovector state would be expected to be close in mass to the $2^{++} a_{2}(1320)$ and the $1^{++} a_{1}(1270)$, and to couple strongly to $K \bar{K}$ and $\eta \pi$. In fact an amplitude analysis of the isovector $K \bar{K}$ system produced in the reaction $\pi^{-} p \rightarrow K^{-} K_{s}^{\circ} p$ at 10 $\mathrm{GeV} / \mathrm{c}[22]$ has provided evidence for just such an $\mathrm{S}$-wave enhancement peaking at $\sim 1.3 \mathrm{GeV} / \mathrm{c}^{2}$.

Finally, it should be noted that a similar approximate mass degeneracy exists among the ${ }^{3} P$ non-s $\bar{s}$ isoscalar states (viz. the $f_{2}(1270)$, the $f_{1}(1285)$ and the $f_{0}(\sim$ $1300)$ ), and in the corresponding strange meson triplet (viz. the $K_{2}^{*}(1430)$, the $Q_{A}(\sim$ 1340) and the $\left.K_{0}^{*}(\mathbf{1 3 5 0})\right)$.

\section{$7.3 t^{\prime}$ DEPENDENCE AT THE $f_{2}^{\prime}(\mathbf{1 5 2 5})$}

In the $f_{2}^{\prime}(1525)$ mass region $\left(1.4-1.7 \mathrm{GeV} / \mathrm{c}^{2}\right)$ the $t_{l m}$ moments shown in fig. 17 have also been fit to partial wave amplitudes with the results shown in fig. 22. Again, there are two distinct solutions which can be characterised by larger and smaller $S$ wave contributions shown as diamond and cross symbols, respectively. The $D_{0}$ intensity exhibits approximately exponential $t^{\prime}$ dependence while $D_{+}$appears to flatten or turn over at low $t^{\prime}$, as expected for a production mechanism resulting in one unit of net helicity flip.

The $D_{-}$intensity again shows no clear structure, while the large uncertainties associated with the $S_{0}$ solutions make it impossible to ascertain the nature of their $t^{\prime}$ dependence. 


\section{Conclusions}

The small, but very clean, sample of events produced via the reaction $K^{-} p \rightarrow K_{s}^{\circ} K_{s}^{\circ} \Lambda$ in the present experiment at $11 \mathrm{GeV} / \mathrm{c}$ incident momentum has yielded several results of relevance to $s \bar{s}$ and gluonium spectroscopy.

(i) The small cluster of events having $M_{K}: K^{\circ}<1.03 \mathrm{GeV} / \mathrm{c}^{2}$ seems consistent with the complex pole structure just below threshold described in ref. 3 , although a conventional interpretation as either an $f_{0}(975)$ or $a_{0}(980)$ meson cannot be ruled out.

(ii) There is no evidence for the production of $f_{2}(\mathbf{1 7 2 0})$, and the upper limit on the cross section times branching fraction to $K \bar{K}$ is $94 n b$ at the $95 \%$ confidence level.

(iii) The $K_{s}^{\circ} K_{s}^{\circ}$ mass spectrum around $2.2 \mathrm{GeV} / \mathrm{c}^{2}$ exhibits structure very similar to that observed in radiative $J / \psi$ decay, and the distribution in $\cos \theta_{J}$ indicates that the associated spin is $\geq 2$. These results together with those of refs. 15 and 16 suggest that the $X(2220)$ may simply be the $2^{++}$or $4^{++}$, mainly $s \bar{s}$, state expected from the quark model.

(iv) The cross section, mass and width of the $f_{2}^{\prime}(1525)$ obtained from the fit to the mass spectrum are consistent with those from other experiments.

(v) An amplitude analysis of the spherical harmonic moments of the $K_{s}^{\circ} K_{s}^{\circ}$ system in the $\mathrm{t}$-channel helicity frame reveals that $f_{2}^{\prime}(1525)$ production via natural parity exchange is $\sim 1.5$ times as strong as helicity zero production via unnatural parity exchange. However the sum of these contributions amounts to only $\sim 66 \%$ of the $f_{2}^{\prime}(1525)$ cross section obtained from the fit to the mass spectrum.

(vi) The cross section difference noted above is due to a structureless $D_{-}$contribution $(\sim 10 \%)$ and an S-wave component $(\sim 25 \%)$ which may correspond to a $0^{++}$ mainly $s \bar{s}$ state approximately degenerate with the $f_{2}^{\prime}(1525)$.

(vii) The $D_{+}$and $D_{0}$ intensities in the $f_{2}^{\prime}(1525)$ region show the difference in $t^{\prime} \mathrm{de}-$ pendence expected on the basis of the production mechanisms involved.

The authors are indebted to the technical staffs of SLAC Group B and the High Energy Physics Laboratory at Nagoya University. Special thanks are due to R. Bierce and M. Takahashi for their excellent supervision of the data processing. 


\section{REFERENCES}

1. D. Aston et al., "The LASS Spectrometer", SLAC-REP-298 (1986).

2. Particle Data Group, Review of Particle Properties, Phys. Lett. 170B, (1986) 1 , and references therein.

3. K.L. Au, D. Morgan and M.R. Pennington, Phys. Rev. D35 (1987) 1633

4. P. Estabrooks, Phys. Rev. D19 (1979) 2678

R. L. Jaffe, Phys. Rev. D15 (1977) 267

D. Robson, Nucl. Phys. B130 (1977) 328

5. C. Edwards et al., Phys. Rev. Lett. 48 (1982) 458

R. M. Baltrusaitis et al., Phys. Rev. D35 (1987) 2077

J.E. Augustin et al., LAL 85-27 (1985)

M. E. B. Franklin, Stanford Ph.D. thesis, SLAC-REP-254 (1982)

6. R. M. Baltrusaitis et al., Phys. Rev. Lett. 56 (1986) 107

7. G. Aiken et al., SLAC-PUB-2642 (1980)

8. M. Aguilar-Benitez et al., Z. Phys. C8 (1981) 313

9. F. Barreiro et al., Nucl. Phys. B121 (1977) 237

10. G. Grayer et al., Nucl. Phys. B75 (1974) 189

11. S.M. Flatté, Phys. Lett. 63B (1976) 224

12. B. V. Bolonkin et al., Sov. J. Nucl. Phys. 43 (1986) 776

13. M. Aguilar-Benitez et al., Phys. Rev. D6 (1972) 29

I. Videau et al., Phys. Lett. 41B (1972) 213

J. Mott et al., Phys. Rev. 177 (1969) 1966

R. Ammar et al., Phys. Rev. Lett. 19 (1967) 1071

D. G. Scotter et al., Nuovo Cimento 62A (1969) 1057

S. Al-Harran et al., Nucl. Phys. B191 (1981) 26

P. Girtler et al., Nucl. Phys. B159 (1979) 397

C. G. Wohl et al., Nucl. Phys. B132 (1978) 401 
14. C. Evangelista et al., Nucl. Phys. B127 (1977) 384

G. W. Brandenburg et al., Nucl. Phys. B104 (1976) 413

T. Armstrong et al., Nucl. Phys. B224 (1983) 193

15. D. Aston et al., SLAC-PUB-4202 (1987)

16. D. Alde et al., Phys. Lett. 177B (1986) 120

17. S. Godfrey and N. Isgur, Phys. Rev. D32 (1985) 189

18. M. Baubillier et al., Z. Phys C17 (1983) 309

19. D. Aston et al., SLAC-PUB-4394, submitted to Phys. Lett. B.

20. Ph. Gavillet et al., Z. Phys C16 (1982) 119

21. J. Weinstein and N. Isgur, Phys. Rev. Lett. 48 (1982) 659;

Phys. Rev. D27 (1983) 588

22. A.D. Martin et al., Phys. Lett. 74B (1978) 417 


\section{FIGURE CAPTIONS}

1. A plan view of the LASS Spectrometer. See ref. 1 for details

2. (a) The $\pi^{+} \pi^{-}$invariant mass distribution of $K_{g}^{\circ}$ daughters and (b) the $\pi^{-} p$ invariant mass of $\boldsymbol{\Lambda}$ daughters for all events with a converged, geometrically constrained fit; (c) and (d) show the $K_{s}^{\circ}$ and $\Lambda$ mass distributions for events in the final data sample (points) compared with those for Monte Carlo events (histograms).

3. A comparison of the measured proper time distribution with Monte Carlo results for (a) $K_{s}^{\circ}$ and (b) $\Lambda$ vertices after the kinematically constrained fit; each data point represents the ratio of the observed to Monte Carlo distribution within the relevant proper time interval.

4. A comparison of the results of the geometrically constrained fit to events in the final data sample (points) with those from Monte Carlo events (histograms): (a) the missing longitudinal momentum, (b) the missing mass squared opposite $K_{s}^{\circ} K_{s}^{\circ} \Lambda$, and (c) the missing mass opposite $K_{s}^{\circ} K_{s}^{\circ}$.

5. (a) The distribution of missing transverse momentum, $\Delta p_{T}$, for the the final data sample (points) and the corresponding Monte Carlo sample (histogram). (b) The $\Delta p_{T}$ distribution for Monte Carlo events generated according to the $K_{s}^{\circ} K_{s}^{\circ} \Sigma^{\circ}$ final state; the open histogram shows the distribution for all events with a geometrically constrained fit to $K_{8}^{\circ} K_{s}^{\circ} \Lambda$, and the shaded histogram shows the distribution for those events which pass the final data selection criteria; note that the vertical scale of (b) has been exaggerated by a factor of 5 .

6. The uncorrected $K_{s}^{\circ} K_{s}^{\circ}$ invariant mass spectrum for the final data sample.

7. The $K_{s}^{\circ} \Lambda$ invariant mass distribution (two entries per event) for the final data sample; the shaded region corresponds to the lower mass combination only.

8. The Dalitz plot for the $K_{s}^{\circ} K_{s}^{\circ} \Lambda$ final data sample; both $K_{s}^{\circ} \Lambda$ mass combinations are plotted for each event.

9. The Chew-Low plot for the final data sample, prior to the requirement $t^{\prime} \leq 2(\mathrm{GeV} / \mathrm{c})^{2}$. 
10. The $\Lambda$ polarization as a function of (a) $M_{K_{\bullet}} K_{\bullet}^{\circ}$, and (b) $t^{\prime}$ for events in the $f_{2}^{\prime}(1525)$ region.

11. The scatter-plots of $t$-channel helicity angle versus $K_{s}^{\circ} K_{s}^{\circ}$ invariant mass: (a) the distribution of the $\operatorname{cosine}$ of the Jackson angle, $\cos \theta_{J}$; and (b) that of the Treiman-Yang angle, $\phi_{T Y}$.

12. The acceptance-corrected $K_{s}^{\circ} K_{s}^{\circ}$ mass distribution in $50 \mathrm{MeV} / \mathrm{c}^{2}$ bins; the threshold region is shown in $10 \mathrm{MeV} / \mathrm{c}^{2}$ bins in the inset; the curve results from a fit using incoherent Breit-Wigner shapes for the $f_{2}(1270)$ and $f_{2}^{\prime}(1525)$ as described in the text.

13. A comparison of fig. 12 to the Mark III results from radiative $J / \psi$ decay [6]; the LASS data have been scaled to match with the Mark III data at the $f_{2}^{\prime}(1525)$ peak; the large Mark III signal for the $f_{2}(1720)$ is not present in the LASS data.

14. A fit to the acceptance-corrected mass spectrum for the reaction $K^{-} p \rightarrow K_{s}^{\circ} K_{s}^{\circ} \Lambda$ at beam momenta (a) $4.2 \mathrm{GeV} / \mathrm{c}$ [9], (b) $11.0 \mathrm{GeV} / \mathrm{c}$ (this experiment) and (c) $40.0 \mathrm{GeV} / \mathrm{c}[12]$; the parametrization is discussed in the text.

15. The beam momentum dependence of the cross section for the reaction $K^{-} p \rightarrow f_{2}^{\prime}(1525) \Lambda$; the line joins the results obtained from the reaction $K^{-} p \rightarrow K_{s}^{\circ} K_{s}^{\circ} \Lambda$ at $4.2 \mathrm{GeV} / \mathrm{c}$ [9] and $11 \mathrm{GeV} / \mathrm{c}$ (this experiment).

16. The mass dependence of the acceptance-corrected unnormalized moments, $t_{l m}$, of the $K_{s}^{\circ} K_{s}^{\circ}$ angular distribution in the $t$-channel helicity frame.

17. The $t^{\prime}$ dependence of the acceptance-corrected moments, $t_{l m}$, of the $K_{s}^{\circ} K_{s}^{\circ}$ angular distribution in the $t$-channel helicity frame for the $f_{2}^{\prime}(1525)$ region.

18. (a) A comparison of the distribution of fig. 12 to the Mark III results from radiative $J / \psi$ decay [6]; the LASS data have been normalized to the number of Mark III events in the mass region $1.8-2.7 \mathrm{GeV} / \mathrm{c}^{2}$; (b) the distribution of $\cos \theta_{J}$ in the 2.0 to $2.4 \mathrm{GeV} / \mathrm{c}^{2}$ mass region (open histogram) and in the 2.15 to 2.3 $\mathrm{GeV} / \mathrm{c}^{2}$ region (hatched histogram).

19. The dependence on $M_{K}$ \% of the intensities of the $D$-wave amplitudes in the region below $1.9 \mathrm{GeV} / \mathrm{c}^{2}$; diamond and cross symbols indicate the solutions which 
give larger and smaller $S$-wave contributions, respectively; the Breit-Wigner curve in (b) is described in the text.

20. (a) The $S$-wave intensity distribution for $M_{K ; K}$; $\leq 1.9 \mathrm{GeV} / \mathrm{c}^{2}$; diamond and cross symbols indicate the larger and smaller $S$-wave solutions, respectively; (b) the $S$-wave intensity distribution as a function of $M_{K^{+} K^{-}}$from the reaction $K^{-} p \rightarrow K^{+} K^{-} \Lambda$ at $8.25 \mathrm{GeV} / \mathrm{c}[18]$; the contributions from the $\Lambda$ helicity-flip and non-flip amplitudes have been summed.

21. The $\chi^{2}$ surface for a single-bin fit in the mass region $1.4-1.7 \mathrm{GeV} / \mathrm{c}^{2}$ plotted as a function of $\left|S_{0}\right|$ and $\left|S_{0}\right|^{2}$ in (a) and (b), respectively; the arrows in the figures indicate the positions of the minima. The $\chi^{2}$ surface is approximately parabolic in $\left|S_{0}\right|$ in the vicinity of each minimum, and its shape is typical for those mass intervals in which the fit yields ambiguous solutions.

22. The $t^{\prime}$ dependence of the $S$ and $D$ wave amplitudes in the $f_{2}^{\prime}(1525)$ region, 1.4-1.7 $\mathrm{GeV} / \mathrm{c}^{2}$; the diamonds and crosses show the larger and smaller $S$ wave solutions, respectively. 